\title{
The Characteristics of Growth of Bacilli Formed Fouling on Wooden Constructions
}

\author{
Volodyslav Yu. Basarab ${ }^{1, a}$, Olga S. Voronkova ${ }^{1, b} *$, Yuliia S. Voronkova ${ }^{2, c}$, \\ Olena V. Severynovska,
} ${ }^{1}$ The Department of Microbiology, Virology and Biotechnology, Oles Honchar Dnipro National
University, Gagarin ave., 72, Dnipro, Ukraine - 49010

${ }^{2}$ The Department of Biochemistry and Biophysics, Oles Honchar Dnipro National University, Gagarin ave., 72, Dnipro, Ukraine - 49010

${ }^{3}$ The Department of Physiology of Human and Animal, Oles Honchar Dnipro National University, Gagarin ave., 72, Dnipro, Ukraine - 49010

abasikc7@gmail.com, b*voronkova_olga@i.ua, cvoronkova1983@gmail.com, deseverinovskaya@gmail.com

Keywords: Bacillus subtilis, biofilm formation, wooden construction.

\begin{abstract}
Problem of biofilm formation have a great significance for environmental microbiological research. Biospheric microorganisms can form biofilm, that provide bacteria resistance to influence of different environmental factors. Some of the most common bacteria in biosphere are bacilli, among them there are film-forming strains. Bacillus spp. is a well-known film forming microorganisms that colonize environmental objects. The biofilm fouling of underwater elements of small wooden constructions located on the Dnieper River near the city of the Dnipro (Ukraine) was studied. It was found that biofilms from surfaces of water constructions include bacilli. It is established that the mean values of CFU in samples from running and still water were $(1.81 \pm 0.52) \times 10^{8}$ and $(1.83 \pm 0.53) \times 10^{8} \mathrm{CFU} / \mathrm{ml}$ respectively per area of wooden sample approximately $1 \mathrm{~cm}^{2}$, while during the laboratory cultivation of the film, formed by these cultures on the plate, the number of cells was $(4.90 \pm 0.93) \times 10^{7}$ and $(4.60 \pm 1.07) \times 10^{7} \mathrm{CFU} / \mathrm{ml}$ per $1 \mathrm{~cm}^{2}$ of the well's bottom, which was an approximate limit of the content of cells of the Bacillus spp. film per unit of area.
\end{abstract}

\section{Introduction}

Nowadays one of the most significant problems in microbiology is the biofilm formation. Usually biofilms are important for clinical bacteriology, because the role of film organization of bacteria in the development of various types of pathology is clearly defined now $[7,12,13]$. At the same time, biofilm is objects of interest of scientists not only because it often determine the pathogenicity of microorganisms.

In addition to clinical role, biofilms are interesting for evolutionary studies: these are the highest forms of interaction of unicellular organisms, in some sense - intermediate forms between unicellular and multicellular forms $[1,6]$. In biofilms, even examples of localized cell death that resemble apoptosis can be observed [17], that allow to form a very specific architectonics.

Biofilms in the biosphere are the main form of microorganisms living. Joint existence provides a number of advantages in the struggle for survival $[2,15,27]$. Among such films are such that formed by bacilli, associated with plant organisms [23]. That biofilms can play a role in plant diseases [27] and in the protection of plants against diseases [15], and may also be dependent in their development on plant-produced compounds [19].

Biospheric biofilms are most often found on the surfaces of water constructions, on the interface between the solid and liquid phases and exist there in conditions of the flow of a medium of varying intensity. The biofilms of such water constructions as cooling systems of power plants, equipment of industrial enterprises, biofilms of treatment facilities, fouling of swimming equipment and bridge constructions are most known. Such biofilms can include not only microorganisms, but also macroobjects, which allows achieving a high level of species diversity $[1,4,10]$. 
The microbial component of biofilms is very variable: it can be either a monoculture or a polymicrobial. A prevalence variant of biofilm is usually monomicrobic form, since it is known that high products have communities with a simplified structure and low diversity $[1,16]$. So, the higher the variety and the complexity, the more energy is required by such system to maintain it, so there is an "energy limit of the complexity of the system" [10]. The complexity of the biotic part of the ecosystem should be limited by the productivity of the community. In addition, the diversity of communities is closely linked to the biotopic and habitat diversity.

Formally, in the techno-ecosystem there is a certain increase in the wealth of elements, at least at the expense of simply adding technical. For example, the following fact is given [1]: the use of a natural reservoir of a water source of an enterprise or an energy station leads to the emergence of a system of canals, technical conduits, dams, hydroconstructions etc. In general, this can cause a significant increase of the biotopic diversity of the entire technoecosystem. But in technoecosystems during the increase of the number of system elements the variety does not increase, but can even decrease due to the sharp dominance of one technogenic factor $[1,4,16]$. In turn, this requires an adaptive reactions from colonists of the surface, and will depend on the adaptive capabilities of the particular microorganism.

In this sense, bacilli are one of the most adaptable bacteria, able to develop during the influence of various environmental factors [11, 20, 24]. Among the film forming bacilli, one of the most famous is Bacillus subtilis, which is a typical biosphere species [9, 25]. Films formed by these bacteria are found on a wide variety of surfaces [9].

The aim of the research was to analyse quantitative characteristics of bacilli growth during formation of biofilm.

\section{Materials and Methods}

For the experiments the fragments of biofouling were taken from wooden constructions of fishing pits located in the main stream of the Dnieper River ( $n=5$, samples 1-5) and in the still water of the Dnieper River ( $\mathrm{n}=5$, samples 6-10) near the city of Dnipro (Ukraine). There are no runoffs of industrial or household wastewater near the points where the samples collected. The samples were collected in the period of May 2017.

Prior to screening, the film was determined visually as a slippery fouling of the surface of a wooden structure in the underwater part. A fragment of the surface (approximately $1 \times 2 \mathrm{~cm}$ in size) with the film was separated by a knife and transferred to a sterile saline solution $(\mathrm{v}=50 \mathrm{ml}, \mathrm{NaCl}$ content $0.5 \%$ ).

The flasks with the samples were placed on a strainer and held there for 10 minutes at $100 \mathrm{rpm}$ to separate the cells. After the end of the buckling, the $10 \mathrm{ml}$ of slurry was taken and a number of dilutions $\left(\begin{array}{llll}10^{-1} & \ldots & 10^{-8}\end{array}\right)$ were prepared. From all of the dilutions the $0.05 \mathrm{ml}$ were inoculated on Petri dishes to obtain pure cultures of microorganisms and make an analysis of the quantity of microorganisms in primary material. Each sample were inoculated thrice on a nutrient medium (meat-peptonic agar, HiMedia, India).

Pure cultures were identified using tinctorial, morphological, cultural and biochemical methods. Identification of isolated bacilli was carried out in accordance with the features of bacteria, listed in Bergey's manual of determinative bacteriology [13].

Identification of bacilli was based on accordance of cultures to next criteries: gram-positive bacilli with ellipsoidal subterminal spores, able to move; positive reaction in tests of catalase production, nitrate reduction, in the Voges-Proskauer test; culture hydrolyzes O-Nitrophenyl- $\beta$-Dgalactopyranoside, utilize citrate, hydrolyze gelatin; grow in the presence of glucose, arabinose and fructose; do not growth on medium with rhamnose; do not product urease, indole and lecithinase; able to hemolysis; colonies are colourless. The culture is prone to overgrowth with the formation of a wrinkled surface. Such cultures were defined as Bacillus spp. [18, 21].

The ability of bacilli strains to form biofilm was determined with use of method of cultivation on plastic plates (TPP, Switzerland). The following quantitative ratio of microorganisms and culture medium were used: suspension of the daily culture of the studied strains $(50 \mu \mathrm{L}$ with $1 \times$ 
Region, Ukraine

$10^{4} \mathrm{CFU} / \mathrm{mL}$ cells $)$ injected into the well with meat-peptonic broth $(200 \mu \mathrm{L})$. Culture incubated in a moist chamber for $72 \mathrm{~h}$ with addition of culture medium if it necessary. The ability to form biofilm determine after removing of medium remnants: the film hung on the walls of plate cell considered as positive response [12].

For determine of the quantitative characteristics of the growth on the plate, after removal of the residues of the nutrient medium, a saline solution was added ( $\mathrm{v}=250 \mu \mathrm{l}, \mathrm{NaCl}$ content $0.5 \%$ ). From the bottom and walls of the 4 well the film was carefully removed by loop and transferred to a glass homogenizer, then adding saline solution to $1 \mathrm{ml}$. The film was destroyed and inoculated on meat-peptonic agar from dilutions of $10^{-2} \ldots 10^{-5}$.

For determine of the spore count in 72-hours film formed on the plate, after removal of the residues of the nutrient medium, a saline solution was added ( $\mathrm{v}=250 \mu \mathrm{l}, \mathrm{NaCl}$ content $0.5 \%)$. From the bottom and walls of the 4 well the film was carefully removed by loop and transferred to a glass homogenizer, then adding saline solution to $1 \mathrm{ml}$. Then suspension heated on the water bath at $70{ }^{\circ} \mathrm{C}$ for $30 \mathrm{~min}$ and made the dilutions of $10^{-2} \ldots 10^{-5}$. Petri dishes with meat-peptonic agar, inoculated by $0.1 \mathrm{ml}$ of suspension from different dilutions, incubated at $29^{\circ} \mathrm{C}$ for $24 \mathrm{~h} \mathrm{[3]}$.

Statistical processing of the results was carried out with use of the program OriginLab 7.5 Pro.

\section{Results and Discussion}

The growth of primary material, obtained by the shaking of samples, showed that in all cases there were more than $10^{8}$ cells on the cut surface of the material from the wooden construction (Table 1). This number is approximate, since it can be assumed that a certain number of cells remained attached to the surface of the sample.

Table 1. Colony forming units on surfaces of samples of wooden constructions, located in the running and still water (number per $\mathrm{cm}^{2}$ of area of sample surface)

\begin{tabular}{|c|c|}
\hline Number of the sample & Cell count, CFU $/ \mathrm{cm}^{2}$ \\
\hline \multicolumn{2}{|c|}{ Samples from areas with running water } \\
\hline 1 & $(1.30 \pm 0.10) \times 10^{8}$ \\
\hline 2 & $(1.27 \pm 0.28) \times 10^{8}$ \\
\hline 3 & $(2.33 \pm 0.15) \times 10^{8}$ \\
\hline 4 & $(1.83 \pm 0.16) \times 10^{8}$ \\
\hline 5 & $(2.33 \pm 0.23) \times 10^{8}$ \\
\hline \multicolumn{2}{|c|}{ Samples from areas with still water } \\
\hline 6 & $(1.77 \pm 0.45) \times 10^{8}$ \\
\hline 7 & $(2.63 \pm 0.28) \times 10^{8}$ \\
\hline 8 & $(1.52 \pm 0.12) \times 10^{8}$ \\
\hline 9 & $(1.23 \pm 0.21) \times 10^{8}$ \\
\hline 10 & $(2.00 \pm 0.13) \times 10^{8}$ \\
\hline
\end{tabular}

Regardless of the point of the sample collection (running or still water), the number of cells had no statistical differences. This may indicate the presence of certain patterns of growth on the underwater elements of construction. The mean values obtained for samples from running and still water were, respectively $(1.81 \pm 0.52) \times 10^{8}$ and $(1.83 \pm 0.53) \times 10^{8} \mathrm{CFU} / \mathrm{ml}$, which is almost identical and does not allow one to assume the presence of dependence the number of cells in the film from the velocity of the environmental flow.

Analysis of morphology of bacterial growth on the surface of the nutrient medium showed that all the colonies belonged to the same type: colourless, matte, flat, with an uneven edge, up to 5 $\mathrm{mm}$ in diameter. When the incubation was prolonged at $+29{ }^{\circ} \mathrm{C}$ to $48-72 \mathrm{~h}$, growth covered the entire surface of the medium. Growth pattern: solid, colourless, matt with the formation of wrinkles, which corresponds to the typical description of the culture of bacilli $[5,22]$. 
Gram-positive rods (after $24 \mathrm{~h}$ incubation) with ellipsoidal spores (after $48 \mathrm{~h}$ of incubation) were detected by microscopy. Identification of microorganisms from the growing colonies made it possible to establish its belonging to the Bacillus spp., whose representatives are known as filmforming microorganisms $[8,14,20]$.

Colonies of other types were found twice in dilutions of $10^{-2}$ and $10^{-1}$ from samples of still water, which most likely can be caused by contamination of the surrounding water [16].

From each sample the one culture was selected to study the ability to formation of biofilm. The growth in a plate was visualised after $18 \mathrm{~h}$, and the film was looked at after $72 \mathrm{~h}$. In all wells, inoculated by the studied cultures, after removing of the nutrient medium the presence of a biofilm on the bottom and walls was confirm. For two samples, growth was observed on the surface of the medium with formation of a wrinkled structure, which is typical for films formed by some species of bacilli, for example, Bacillus subtilis [22].

A quantitative analysis of the growth on the plate showed that the growth rates of the films have some differences (Table 2).

Table 2. Colony forming units and spore count in 72-h biofilm during laboratory cultivation of cultures, isolated from primary samples (number per $\mathrm{cm}^{2}$ of area of well of plate)

\begin{tabular}{|c|c|c|}
\hline Number of culture* & Cell count, CFU $/ \mathrm{cm}^{2}$ & Spore count, spore $/ \mathrm{cm}^{2}$ \\
\hline \multicolumn{3}{|c|}{ Samples from areas with running water } \\
\hline 1 & $3.73 \times 10^{7}$ & $2.16 \times 10^{6}$ \\
\hline 2 & $5.67 \times 10^{7}$ & $2.99 \times 10^{6}$ \\
\hline 3 & $5.97 \times 10^{7}$ & $1.04 \times 10^{6}$ \\
\hline 4 & $4.33 \times 10^{7}$ & $1.72 \times 10^{6}$ \\
\hline 5 & $4.78 \times 10^{7}$ & $2.39 \times 10^{6}$ \\
\hline \multicolumn{3}{|c|}{ Samples from areas with still water } \\
\hline 6 & $5.37 \times 10^{7}$ & $2.91 \times 10^{6}$ \\
\hline 7 & $4.18 \times 10^{7}$ & $1.64 \times 10^{6}$ \\
\hline 8 & $4.78 \times 10^{7}$ & $1.42 \times 10^{6}$ \\
\hline 9 & $2.99 \times 10^{7}$ & $1.94 \times 10^{6}$ \\
\hline 10 & $5.67 \times 10^{7}$ & $2.09 \times 10^{6}$ \\
\hline
\end{tabular}

Asterisks [*] denotes that the culture number is the same as the sample number, from which it is selected

The mean values of the number of cells in films grown on plates were for cultures obtained from samples with running water $(4.90 \pm 0.93) \times 10^{7}$ and $(4.60 \pm 1.07) \times 10^{7} \mathrm{CFU} / \mathrm{ml}$ in samples from still water. The mean values of spore count for the cultures, isolated from running water, was $(2.60 \pm 0.73) \times 10^{6}$ spore $/ \mathrm{cm}^{2}$, and for samples from still water $-(2.00 \pm 0.57) \times 10^{6} \mathrm{spore} / \mathrm{cm}^{2}$. The ratio of spores/alive cells in biofilm of bacilli cultures was approximately $1 / 20$. A significantly less spore count than alive cells in the 72-hour biofilm can be explained by the removal of residual of nutrient medium, during which part of the spores could be removed.

When comparing the number of cells per unit of area of growth (per $1 \mathrm{~cm}^{2}$ ), it can be concluded that the gain was approximately the similar for films formed in open water sources and grown in the laboratory. It should be noted, that biospheric biofilm has a greater volume of environment for the formation and a constant inflow of nutrients, which is absent in the conditions of the plate growth. In addition, under external conditions, the film forms and exists for a much longer time than during experiments in the laboratory. Part of the cells during the growth of the Bacillus spp. culture die, forming the basis of wrinkles, which are visible with the growth of culture on the surface of solid media [5, 17], which is also practically not achieved in the conditions of short-term $(72 \mathrm{~h})$ cultivation in the plate. That is, a quantitative index of $10^{7}-10^{8} \mathrm{CFU} / \mathrm{ml}$ can be considered a critical value of the number of cells in biofilms formed by this species of bacteria on underwater wooden surfaces in open water sources. 


\section{Conclusion}

It was shown that for underwater elements of small wooden constructions located on the Dnieper River near the city of the Dnipro (Ukraine) is typically a presence of biofouling, formed by bacilli.

Regardless of the point of the material collection (running or still water), the amount of cells in the film was $10^{8} \mathrm{CFU} / \mathrm{ml}$ per $1 \mathrm{~cm}^{2}$ of wooden fragment and more, while in the laboratory cultivation of the film in the plate it is about $10^{7} \mathrm{CFU} / \mathrm{ml}$ per $1 \mathrm{~cm}^{2}$ of the well's bottom, which is an approximate limit of the content of cells of the bacilli film per unit of area in experiment.

\section{Conflict of Interest}

The authors declare that there is no conflict of interest.

\section{References}

[1] A.A. Protasov, Aquatic techno-ecosystems and their place in biosphere, Journal of Siberian Federal University. Biology. 4(6) (2013) 405-423. (In Russian)

[2] A.L. McLoon et al., Tracing the domestication of a biofilm forming bacterium, J. Bacteriol. 193 (2011) 2027-2034.

[3] A.S. Labinskaya, Ye.G. Volina (Eds.), Manual on medical microbiology. General and sanitary microbiology. Book 1, Binom, Moscow, Russia, 2008. (in Russian)

[4] A.Y. Zvyagintsev, O.P. Poltarukha, S.I. Maslennikov, Fouling on technical water supply marine systems and protection method analysis of fouling on water conduits (Analytical review), Voda: Khimiya i Ekologiya. 1 (2015) 30-51. (in Russian)

[5] D. Romero et al., Amyloid fibers provide structural integrity to Bacillus subtilis biofilms, Proc. Natl. Acad. Sci. USA. 107 (2010) 2230-2234.

[6] E.A. Shank, R. Kolter, Extracellular signaling and multicellularity in Bacillus subtilis, Curr. Opin. Microbiol. 14 (2011) 741-747.

[7] E.Y. Trizna et al., Thio derivatives of $2(5 \mathrm{H})$-furanone as inhibitors against Bacillus subtilis biofilms, Acta Naturae. 7(2) (2015) 110-116. (in Russian)

[8] F. Yan et al., The comER gene plays an important role in biofilm formation and sporulation in both Bacillus subtilis and Bacillus cereus, Front. Microbiol. 7 (2016) Article 1025.

[9] H. Vlamakis et al., Sticking together: Building a biofilm the Bacillus subtilis way, Nat. Rev. Microbiol. 11(3) (2013) 157-168.

[10] I.V. Burkovskiy, Marine biogeocenology. Organization of communities and ecosystems, Partnership of Scientific Publications KMK, Moscow, Russia, 2006. (in Russian)

[11] J. Dervaux, J.C. Magniez, A. Libchaber, On growth and form of Bacillus subtilis biofilms, Interface Focus. 4 (2014) 20130051.

[12] J.E. Cassat, M.S. Smeltzer, C.Y. Lee, Investigation of biofilm formation in clinical isolates of Staphylococcus aureus, in: J. Yinduo (Ed.), Methicillin-resistant Staphylococcus aureus (MRSA). Protocols, Methods in Molecular Biology, Humana press, St. Paul, 2014.

[13] J.G. Holt et al. (Eds.), Bergey's manual of determinative bacteriology, Williams \& Wilkins, Baltimore, 1994.

[14] K. Kobayashi, Bacillus subtilis pellicle formation proceeds through genetically defined morphological changes, J. Bacteriol. 189 (13) (2007) 4920-4931. 
[15] L.S. Cairns, L. Hobley, N.R. Stanley-Wall, Biofilm formation by Bacillus subtilis: New insights into regulatory strategies and assembly mechanisms, Mol. Microbiol. 93 (2014) 587598.

[16] L.V. Didenko et al., Morphological features of biofilms in potentially dangerous water systems, Epidemiologiya i infektsionnyye bolezni. 1 (2012) 15-20. (in Russian)

[17] M. Asallya et al., Localized cell death focuses mechanical forces during 3D patterning in a biofilm, PNAS. 109 (46) (2012) 18891-18896.

[18] N.A. Logan, R.C.W. Berkeley, Identification of Bacillus strains using the API system, J. Gen. Microbiol. 130 (1984) 1871-1882.

[19] P.B. Beauregard et al., Bacillus subtilis biofilm induction by plant polysaccharides, Proc. Natl. Acad. Sci. USA. 110 (2013) E1621-E1630.

[20] S. Arnaouteli, C.E. MacPhee, N.R. Stanley-Wall, Just in case it rains: Building a hydrophobic biofilm the Bacillus subtilis way, Curr. Opin. Microbiol. 34 (2016) 7-12.

[21] S. Aryal, Biochemical test and identification of Bacillus subtilis, 2016. Available: www.microbiologyinfo.com/biochemical-test-and- identification-of-bacillus-subtilis/.

[22] S.S. Branda et al., Fruiting body formation by Bacillus subtilis, Proc. Natl. Acad. Sci. USA. 98 (2001) 11621-11626.

[23] T. Gao et al., Alternative modes of biofilm formation by plant-associated Bacillus cereus, Microbiology Open. 4 (2015) 452-464.

[24] W. Ma et al., Bacillus subtilis biofilm development in the presence of soil clay minerals and iron oxides, npj Biofilms and Microbiomes. 3 (2017) 4.

[25] Y. Chai et al., Galactose metabolism plays a crucial role in biofilm formation by Bacillus subtilis, mBio 3(4) (2012) e00184-12.

[26] Y. Chen et al., Biocontrol of tomato wilt disease by Bacillus subtilis isolates from natural environments depends on conserved genes mediating biofilm formation, Environ. Microbiol. 15 (2013) 848-864.

[27] Z. Hong et al., Initial adhesion of Bacillus subtilis on soil minerals as related to their surface properties, Eur. J. Soil Sci. 63 (2012) 457-466. 